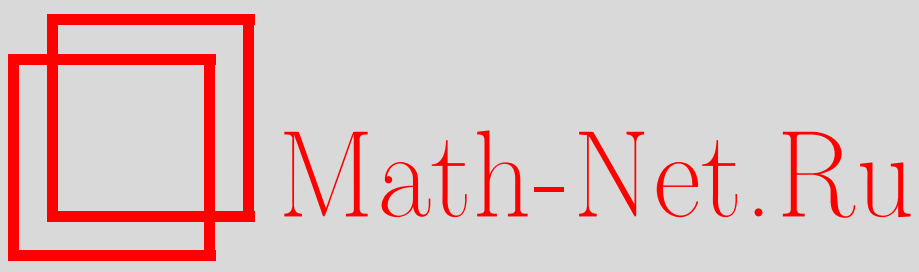

В. В. Кабанов, А. А. Махнев, Об отделимых графах с некоторыми условиями регулярности, Матем. сб., 1996, том 187, номер 10, 73-86

DOI: https://doi.org/10.4213/sm165

Использование Общероссийского математического портала Math-Net.Ru подразумевает, что вы прочитали и согласны с пользовательским соглашением http: //www. mathnet.ru/rus/agreement

Параметры загрузки:

IP: 52.6 .47 .48

26 апреля 2023 г., 15:01:29 
УДК 519.17

\author{
В.В. Кабанов, А. А. Махнев
}

\title{
Об отделимых графах с некоторыми условиями регулярности
}

\begin{abstract}
В работе доказываются две теоремы. В теореме 1 описаны связные $\mu$-регулярные графы без 3 -лап. В теореме 2 получены необходимые и достаточные условия для того, чтобы связный вполне регулярный граф̆ с $\mu>1$ был отделим. Отделимым мы называем граф $\Gamma$, у которого для любой вершины $a$ из $\Gamma$ подграб $\Gamma_{2}(a)$ содержит вершины $b, c$ на расстоянии 2 в $\Gamma_{2}(a)$, и $\mu$-подграф̆ для любой такой пары не пересекает окрестность вершины $a$.

Библиографофия: 4 названия.
\end{abstract}

\section{§1. Введение}

Мы рассматриваем неориентированные графы без петель и кратных ребер. Если $a, b$ вершины графа $\Gamma$, то через $d(a, b)$ обозначим расстояние между $a$ и $b$, а через $\Gamma_{i}(a)$ обозначим подграф, индуцированный $\Gamma$ на множестве всех вершин графа $\Gamma$, которые находятся на расстоянии $i$ от вершины $a$. Заметим, что всюду далее, если $\Delta$ - подмножество из множества вершин графа $\Gamma$, то подграф $\Delta$ из $\Gamma$ - это индуцированный подграф графа $\Gamma$ на $\Delta$. Подграф $\Gamma_{1}(a)$ мы будем называть окрестностью вериинь $а$ и обозначать через $[a]$. Через $a^{\perp}$ обозначим подграф̆ на множестве $\{a\} \cup[a]$.

Граф Г называется регулярным валентности $k$, если $[a]$ содержит $k$ вершин для любой вершины $a$ из $Г$. Граф Г называется $\mu$-регулярныцм графом с параметрами $(v, k, \mu)$, если $|\Gamma|=v$, граф $Г$ регулярен валентности $k$ и каждая пара вершин из $\Gamma$, которые находятся на расстоянии 2 в $\Gamma$, имеет $\mu$ общих смежных с ними вершин. Более того, подграф̆ на этих $\mu$ вершинах будем называть $\mu$-подграфом. Граф $\Gamma$ на $v$ вершинах назовем реберно регулярным с параметрами $(v, k, \lambda)$, если он регулярен валентности $k$ и каждое ребро лежит в $\lambda$ треугольниках. Граф̆ $\Gamma$ - вполне регулярный граф с параметрами $(v, k, \lambda, \mu)$, если он $\mu$-регулярен и реберно регулярен с соответствуюшими параметрами. Вполне регулярный граф называется сильно регулярным графом, если он имеет диаметр 2.

Граф Тервиллигера - это неполный граф Г такой, что для любых двух вершин $a, b$ на расстоянии 2 подграф $[a] \cap[b]$ является кликой порядка $\mu$ для некоторого фиксированного $\mu \geqslant 0$.

Через $\left(m_{1}, \ldots, m_{n}\right)$ обозначим полный многодольный граф̆, с долями порядка $m_{1}, \ldots, m_{n}$. Граф $(m, 1)$ назьвается $m$-лапой, если $m \geqslant 2$. Граф на множестве $X_{1} \times \cdots \times X_{n}$ назьвается $m_{1} \times \cdots \times m_{n}$-графом, если $\left|X_{i}\right|=m_{i}$, апары $\left(x_{1}, \ldots, x_{n}\right)$

Работа выполнена при финансовой поддержке Российского фонда фундаментальных исследований (гранты № 93-01-01529 и № 94-01-00802-а). 
и $\left(y_{1}, \ldots, y_{n}\right)$ смежны тогда и только тогда, когда существует единственное $i$ такое, что $x_{i} \neq y_{i}$. При $m_{i}=m$, для всех $i$ этот граф называется графом Хемминга $H(n, m)$ или $n$-мерным решетчатылм графом. Треугольным графом $T(m)$ называется граф̆ с множеством неупорядоченных пар из $X$ в качестве вершин, $|X|=m$ и пары $\{a, b\},\{c, d\}$ смежны тогда и только тогда, ког да они имеют общий элемент. Антиподальный граф диаметра 3 на 12 вершинах, у которого окрестность любой вершины - пятиугольник, назовем графом икосаэдра.

Под $\alpha$-расширением графа Г будем понимать граф, полученный заменой каждой вершины $a$ из Г на полный подграф $(a)$, содержаший $\alpha$ вершин (такой подграф называется $\alpha$-кликой), причем вершины из $(a)$ и $(b)$ смежны тогда и только тогда, когда $a$ и $b$ смежны в $Г$. Для вершины $a$ регулярного графа $\Gamma$ ядро $K(a)-$ это множество $\left\{x \in \Gamma \mid x^{\perp}=a^{\perp}\right\}$.

Пусть $\mathscr{F}$ некоторый класс графов. Граф Г называется локально $\mathscr{F}$-графом, если окрестность каждой вершины из Г принадлежит $\mathscr{F}$. Большое число исследований было посвящено изучению свойств локально $\mathscr{F}$-графов при различных ограничениях на класс графов $\mathscr{F}$. Обзор этих работ можно найти, например, в [4]. Известно строение локально $m \times 2$-графов [4, теорема 9.1.3]. Очевидно, что такие графы не содержат 3-коклик в окрестности любой вершины. Теорема 1 данной статьи посвящена классификации $\mu$-регулярных локально $\mathscr{F}$-графов, где $\mathscr{F}-$ класс графов не содержащих 3-коклик. Заметим, что в этом случае сам граф̆ не содержит 3-лап. Важным вкладом в изучение этого класса графов явилась классификация Зейделем [4, теорема 3.12.4] сильно регулярных графов без 3-лап. В работе [1] получено описание $\mu$-регулярных графов диаметра 2 без 3-лап. Теорема 1 снимает ограничение на диаметр графа.

Теорема 1. Пусть Г - связный $\mu$-регулярный граф без 3-лап. Тогда Г либо не содержит 3-коклик, либо является $\alpha$-расширением одного из следующих графов:

(1) регулярного графа Тервиллигера с $\mu=1$ диаметра $>2$;

(2) прямоугольной решетки $m \times n$ при $m \geqslant 3, n \geqslant 3$;

(3) треугольного графа $T(m)$ nри $m \geqslant 6$;

(4) графа ШІлефли (дополнительного $к$ точечному графу обобщенного четырехугольника $G Q(2,4))$;

(5) графа икосаэдра.

Примеры регулярных графов без 3-лап имеются среди графов вершин и ребер полуправильных многогранников. Граф усеченного тетраэдра (рис. 1) является $\mu$-регулярным с $\mu=1$, но не реберно регулярным графом. Граф кубооктаэдра (рис. 2), наоборот, реберно регулярен, но не является $\mu$-регулярньм.

Граф̆ Г назовем отделимылм, если для любой вершины $a$ из $Г$ подграф̆ $\Gamma_{2}(a)$ содержит вершины $b, c$ на расстоянии 2 в $\Gamma_{2}(a)$, и $\mu$-подграф $[b] \cap[c]$ для любой такой пары не пересекает $[a]$.

Для любого графа Г с 4-циклом, удовлетворяющего условиям теоремы 1, имеет место альтернатива: либо граф Г не содержит 3-коклик, либо граф Г отделим. Результат, полученный в теореме 1 , позволяет описать вполне регулярные отделимые графы.

ТЕОРЕМА 2. Пусть Г - связный вполне регулярный граф с параметрами $(v, k, \lambda, \mu), \mu>1$. Граф Г отделим тогда и только тогда, когда он является 


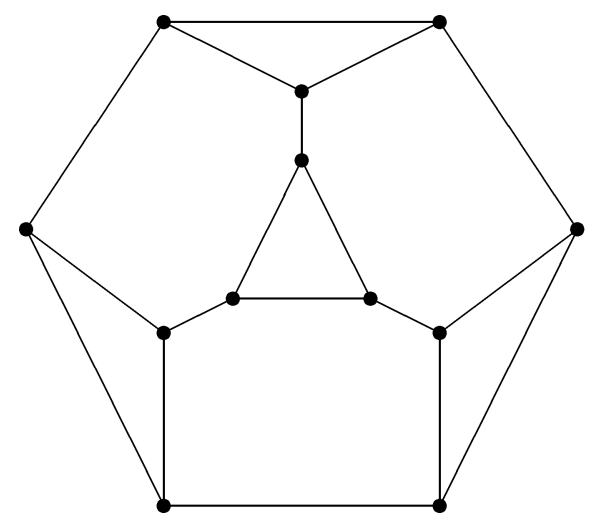

Рис. 1. Граф усеченного тетраэдра

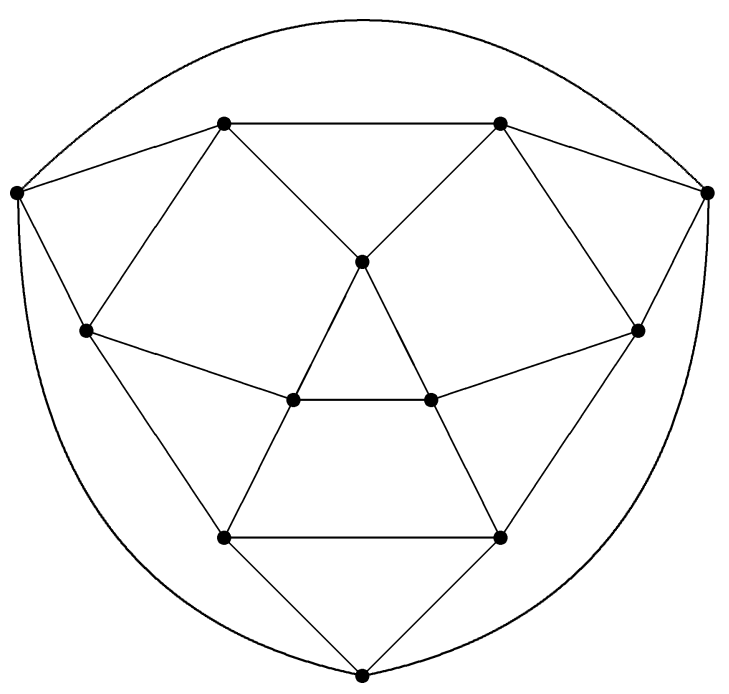

Рис. 2. Граф кубооктаэдра

одним из следующих графов:

(1) граф Хемминга $H(n, m)$ nри $m \geqslant 3, n \geqslant 2$;

(2) треугольный граф $T(m)$ при $m \geqslant 6$;

(3) граф Шлефли;

(4) граф икосаэдра.

Некоторые свойства регулярных отделимых графов получены в $\S 2$. В $\S 3$ рассматриваются регулярные отделимые графы с 3-лапами, в которых любой $\mu$-подграф̆ содержит не менее двух вершин. В $\S 4$ проведена редукция $\mu$-регулярных гра- 
фов без 3-лап к графам Тервиллигера. Параграфы 5 и 6 посвяшены классификации графов Тервиллигера без 3-лап.

\section{§ 2. О регулярных отделимых графах}

В этом параграфе $\Gamma$ - связный регулярный граф. Мы предполагаем, что для любой вершины $a$ из граф̆а $Г$ подграф̆ $\Gamma_{2}(a)$ содержит вершины $b, c$ на расстоянии 2 в $\Gamma_{2}(a)$, и для любых двух таких вершин $[b] \cap[c]$ не пересекает $[a]$.

Лемма 2.1. Если аd - ребро из графа $\Gamma$, то подграф $[a]-[d]$ не содерәсит вершин, находящихся на расстоянии 2 в $\Gamma_{2}(d)$. В частности, он является обвединением изолированных клик.

ДоказАТЕльство. Пусть $b, c$ - вершины из $[a]-[d]$, находящиеся на расстоянии 2 в $\Gamma_{2}(d)$. По условию $[b] \cap[c]$ не пересекает $[d]$. Противоречие с тем, что $[b] \cap[c]$ содержит вершину $a$ из $[d]$.

Лемма 2.2. Пусть $d(a, b)=2 u c \in[a] \cap[b]$.

(1) Еслие, с-смежсные вершины из $[a] \cap[b]$, то $[e]-[c] u[c]-[e]$ содержатся $\boldsymbol{\theta}[a] \cup[b]$.

(2) Если $w \in[c]-\left(a^{\perp} \cup b^{\perp}\right)$, то содержсащая вериину с связная компонента из $\mu$-подграфа $[a] \cap[b]$ лежсит в $[w]$.

(3) Подграф $([c]-[a]) \cap[b]$ является кликой.

ДоказАтельство. (1) Допустим, что $[c]-[e]$ содержит вершину $d$ не лежашую в $[a] \cup[b]$. Тогда $\Gamma_{2}(d)$ содержит 2 -лапу $\{e ; a, b\}$. Противоречие с тем, что $[a] \cap[b]$ содержит вершину $c$ из $[d]$.

(2) Достаточно доказать, что $[w]$ содержит любую смежную с $c$ вершину $d$ из $[a] \cap[b]$. Но если $d \notin[w]$, то $\{d ; a, b\}$ будет 2-лапой из $\Gamma_{2}(w)$.

(3) По лемме $2.1[c]-a^{\perp}$ является объединением изолированных клик, в одной из которых лежит $b$.

ЛЕмма 2.3. Пусть а, с-смежсные вериины из $\Gamma u[a] \cap[c]$ содержит 3-коклику $\{d, e, f\}$. Тогда содержсащая а связная компонента $\Lambda$ из $[d] \cap[e] \cap[f]$ совпадает с $K($ a).

ДокаЗАТЕЛьство. Положим $\Delta=\bigcap_{x \in \Lambda}[x]$. Из леммы $2.2(2)$ следует, что $\Lambda-$ связная компонента из $\mu$-подграф̆а $[y] \cap[z]$ для любых двух вершин $y, z$ из $\{d, e, f\}$. В частности, $[y] \cap[z]$ не пересекает $\Delta$.

По лемме $2.2(1)$ каждая вершина $x$ из $[a]-[c]$ смежна по крайней мере с двумя вершинами из $\{d, e, f\}$, поэтому $[a]-[c]$ и $[c]-[a]$ лежат в $\Lambda$. Покажем, что $[x]$ лежит в $\Lambda \cup \Delta$ для любой вершины $x$ из $\Lambda$. Для этого достаточно показать, что $[a] \cap[c] \subset \Lambda \cup \Delta$. Пусть $g \in[a] \cap[c]-\Lambda$ и $g$ не смежна с некоторой вершиной $w$ из $\Lambda$. Выберем $a=w_{0}, w_{1}, \ldots, w_{n}=w$-путь в $\Lambda$ и $i$ - наименьший номер такой, что [ $\left.w_{i}\right]$ не содержит $g$. Как показано выше, $\left[w_{i-1}\right]-\left[w_{i}\right] \subset \Lambda$. Противоречие с выбором $g$.

Допустим, что $[c]-[a]$ содержит вершину $b$ (ясно, что $b \in \Lambda$ ). Покажем, что в этом случае $\Gamma=\Lambda \cup \Delta$. Для этого достаточно доказать, что $[x] \subset \Lambda \cup \Delta$ для любой вершины $x$ из $\Delta$. Но если $x \in \Delta$ и $g$ - вершина из $[x]$, не лежашая в $\Lambda \cup \Delta$, то $\Gamma_{2}(g)$ содержит 2-лапу $\{c ; a, b\}$, причем $[a] \cap[b]$ содержит вершину $x$ из $[g]$. Противоречие. 
Итак, либо $\Lambda=K(a)$, либо $\Gamma=\Lambda \cup \Delta$. Но в последнем случае по условию $\Gamma_{2}(a)=\Lambda-a^{\perp}$ содержит 2-лапу $\{w ; x, y\}$. Противоречие с тем, что $[x] \cap[y]$ содержит $\Delta$.

ЛЕмма 2.4. Пусть $d(a, b)=2$.

(1) Если $c \in[a] \cap[b]$ и $[c]$ не лежсит в $a^{\perp} \cup b^{\perp}$, то содерәсащая с связная компонента из $[a] \cap[b]$ совпадает с $K(c)$.

(2) Eсли $[a] \cap[b]$ содержит ребро cd u $c \notin K(d)$, mo $[c] u[d]$ лежсат в $a^{\perp} \cup b^{\perp}$.

ДокАЗАТЕльство. Первое утверждение следует из леммы 2.3, а второе является следствием первого.

Лемма 2.5. Верны следующие утверждения:

(1) если bc-ребро из $\Gamma_{2}(a)$ и $d \in([a] \cap[b])-[c]$, то любая вершина из $[c] \cap[d]$ лехит в $a^{\perp} \cup b^{\perp}$

(2) если аесbd - индуцированный пятиугольник из Г, то любая вершина из $[c] \cap[d]$ лежит в $b^{\perp}$ или смежнна с а ие.

ДоказАтельство. (1) Пусть $[c] \cap[d]$ содержит вершину $w$, не лежащую в $a^{\perp} \cup b^{\perp}$. Тогда 2-лапа $\{c ; b, w\}$ лежит в $\Gamma_{2}(a)$. Противоречие с тем, что $[b] \cap[w]$ содержит вершину $d$ из $[a]$.

(2) Пусть $w \in([c] \cap[d])-b^{\perp}$. Из первого утверждения леммы и симметричности пятиугольника следует, что $w \in[a] \cap[e]$. Лемма доказана.

ЛЕмма 2.6. Если $\{a, b ; c, d, e\}-(2,3)$-подграф из $\Gamma$, то $[y]$ не лехит $\boldsymbol{в}$ $a^{\perp} \cup b^{\perp} u x^{\perp} \cap y^{\perp}=K(x) \cup K(y)$ для любиц $x \in\{a, b\}, y \in\{c, d, e\}$.

ДоказАтельство. Пусть $f \in[a] \cap[c]$. Если $f$ смежна с $d$, то $f$ лежит в содержашей $a$ связной компоненте $\Lambda$ из $[c] \cap[d]$ и по лемме $2.4(1) \Lambda=K(a)$. Допустим, что $f$ не смежна с $d$ и $e$. Если $f \notin[b]$, то $\{b ; d, e\}-2$-лапа из $\Gamma_{2}(f)$. Противоречие с тем, что $[d] \cap[e]$ содержит вершину $а$ из $[f]$. Значит, $[c] \cap[a]-K(a) \subset[b]$. Если $[c]$ не лежит в $a^{\perp} \cup b^{\perp}$, то по лемме 2.4 (1) содержашая $c$ связная компонента из $[a] \cap[b]$ совпадает с $K(c)$. В частности, в этом случае $a^{\perp} \cap c^{\perp}=K(a) \cup K(c)$.

Предположим, что $[c] \subset a^{\perp} \cup b^{\perp}$. По условию $\Gamma_{2}(c)$ содержит 2-лапу $\{z ; u, w\}$. Без ограничения общности, $u \in[a]-[b], w \in[b]-[a]$. Если $z \in[a]-[b]$, то мы получим индуцированный пятиугольник $a c b w z$. По лемме $2.5(2)$ вершина $d$ из $[a] \cap[b]$ смежна с $w$ и $z$. Противоречие с тем, что $([a] \cap[d])-K(a)$ содержится в $[b]$. Итак, $z \in[a] \cap[b]$.

Если $z$ не смежна с вершиной $x$ из $\{e, d\}$, то $\{c, x, z\}$ является 3-кокликой из $[a] \cap[b]$. Повторяя рассуждения из начала доказательства леммы, убедимся, что $([z] \cap[a])-K(a)$ лежит в $[a] \cap[b]$. Если же $z \in[e] \cap[d]$, то $z \in K(a)$. Полученное противоречие доказывает лемму.

\section{§ 3. Отделимые реберно регулярные графы, содержащие 3-лапы}

В этом параграфе мы докажем

ПРЕДЛОЖЕНИЕ 1. Пусть Г - связный отделимый реберно регулярный граф c параметрами $(v, k, \lambda)$, в котором для любых двух вершин $a, b$ на расстоянии 2 в Г подграф $[a] \cap[b]$ содержит не менее двух вериин. Если $Г$ содержит 3-лапу, то Г - граф Хемминга $H(n, m), n \geqslant 3, m \geqslant 3$. 
Пусть Г удовлетворяет условиям предложения 1.

ЛЕмма 3.1. Если $[a]$ содержит 3-коклику, то $[a]$ является обвединением изолированных клик порядка $\lambda+1$.

ДокАЗАтЕльство. Пусть $\{c, d, e\}-3$-коклика из $a^{\perp}$. По лемме $2.4(1)$ связная компонента из $\mu$-подграфа $[c] \cap[d]$, содержашая $a$, совпадает с $K(a)$. Из реберной регулярности графа Г следует, что $K(a)=\{a\}$. Отсюда $c, d$ лежат в изолированных кликах из $[a]$ порядка $\lambda+1$. Далее, любая вершина из $[a]$ лежит в некоторой 3 -коклике из $[a]$.

ЛЕмма 3.2. Если [a] содержит 3-коклику, то окрестность кажсдой вериинь из Г содерэсит 3-коклику. Более того, для любъх двух вериин на расстоянии 2 в Г подграф $[x] \cap[y]$ является 2-кокликой.

ДокАЗАТЕЛЬство. Ввиду связности графа Г достаточно убедиться, что произвольная вершина $c$ из $a^{\perp}$ смежна с некоторой 3 -кокликой. Пусть $c^{\perp}$ не содержит 3-коклик. Тогда $[c]-a^{\perp}$ является кликой, так как $[c] \cap a^{\perp}-$ изолированная $\lambda+1$-клика из $[c]$. Противоречие с тем, что $k-(\lambda+1) \geqslant 2(\lambda+1)$.

Пусть теперь $b \in \Gamma_{2}(a)$ и $[a] \cap[b]$ содержит две различные вершины $c, d$. По лемме $2.4(1)$ каждая из вершин в $[a] \cap[b]$ изолирована в $[a] \cap[b]$. Значит, $[a] \cap[b]$ - коклика. Если $[a] \cap[b]$ содержит более двух вершин, то по лемме $2.6 a^{\perp} \cap c^{\perp}=$ $K(a) \cup K(c)$. Поэтому $\lambda=0$. Таким образом, $Г$ не содержит треугольников. Если $f g$ - ребро из $\Gamma_{2}(a)$, то $[a] \cap[f] \cap[g]$ пусто. Для $x \in[a] \cap[f], y \in[a] \cap[g]$ мы получим пятиугольник $a x f g y$. Противоречие с леммой 2.5 (2). Лемма доказана.

В работе [2] Х. Еномото доказал следующее.

Если Г является вполне регулярным графом с параметрами $(v, k, \lambda, \mu)$, $\mu>1, u$ Г не содержит индуцированных пятиугольников и полных подграфов на четырех вершинах с одним удаленным ребром, то $n=k /(\lambda+1)$ - иелое число,

$$
\left|\Gamma_{i}(a)\right| \leqslant \frac{n !(\lambda+1)^{i}}{i !(n-i) !}=C_{n}^{i}(\lambda+1)^{i}
$$

для любой вериинь а графа $Г и i \geqslant 0$. Более того, Г имеет диаметр $d \leqslant n u$ число вериин $v \leqslant(\lambda+2)^{n}$.

Если $v=(\lambda+2)^{n}$, то $\mu=2$ и Г является графом Хемминга $H(n, \lambda+2)$.

Чтобы завершить доказательство предложения 1 нам потребуется частичное обращение последнего утверждения в случае, когда $\mu=2$.

ПРЕДЛОЖЕНИЕ 2. Пусть Г является вполне регулярным графом с параметрами $(v, k, \lambda, \mu)$ и Г не содержит индуцированных пятиугольников. Если все $\mu$-подграфы из графа $Г$ являются 2-кокликами, то $Г-$ граф Хемминга $H(n, m), n=k /(\lambda+1), m=\lambda+2$.

Пусть граф Г удовлетворяет условиям предложения 2. Так как все $\mu$-подграфы из $\Gamma$ являются 2-кокликами, то окрестность любой вершины в $\Gamma$ - объединение $n$ изолированных клик порядка $\lambda+1$. Пусть $a, b \in \Gamma$ и $d(a, b)=i$.

ЛЕмма 3.3. Подграф $\Gamma_{i}(a) \cap[b]$ является $i$-кокликой. 
ДокАЗАТЕльство. Пусть вершина $c$ принадлежит $\Gamma_{i-1}(a) \cap[b]$. По индукции, $\Gamma_{i-2}(a) \cap[c]=\left\{w_{1}, \ldots, w_{i-1}\right\}$ является $(i-1)$-кокликой.

Так же, как и в [2], рассмотрим подграф

$$
V=\bigcup_{j=1}^{i-1}\left[w_{j}\right] \cap[b]
$$

По лемме 2.2 из работы [2] подграф $V$ содержит $i$-коклику. Поскольку любой $\mu$-подграф̆ $\left[w_{j}\right] \cap[b]$ является 2-кокликой и содержит вершину $c$, то $V$ не может содержать более, чем $i$ вершин. Лемма доказана.

Лемма 3.4. Для любой вериинъь $b$ из $\Gamma_{i}(a) \quad\left|[b] \cap \Gamma_{i+1}(a)\right|=(n-i)(\lambda+1)$.

ДокАЗАТЕЛьство. Пусть $W=\Gamma_{i}(a) \cap[b]=\left\{w_{1}, \ldots, w_{i}\right\}$. Подграф $W$ является $i$-кокликой по лемме 3.3 , а подграфы $\left[w_{j}\right] \cap[b], 1 \leqslant j \leqslant i$, являются $\lambda$-кликами. Так как, $[b] \cap \Gamma_{i+1}(a)$ совпадает с $[b]-\bigcup_{j=1}^{i} w_{j}^{\perp}$, то $\left|[b] \cap \Gamma_{i+1}(a)\right|=k-i(\lambda+1)=$ $(n-i)(\lambda+1)$. Лемма доказана.

Лемма 3.5. Справедливо равенство $v=(\lambda+2)^{n}$.

ДокАЗАтЕльство. Докажем сначала, что $\left|\Gamma_{i}(a)\right|=C_{n}^{i}(\lambda+1)^{i}$, для чего подсчитаем число ребер между $\Gamma_{i}(a)$ и $\Gamma_{i+1}(a)$. По леммам 3.3 и 3.4 мы имеем следуюшее равенство $\left|\Gamma_{i+1}(a)\right| i=\left|\Gamma_{i}(a)\right|(n-i)(\lambda+1)$. Значит, по индукции,

$$
\left|\Gamma_{i+1}(a)\right|=\frac{n !(\lambda+1)^{i+1}}{(i+1) !(n-i-1) !}=C_{n}^{i+1}(\lambda+1)^{i+1}
$$

Но $\Gamma_{i}(a)$ не пересекаются для различных $i$ и, следовательно, мы получили биномиальное разложение для числа вершин графа Г. Лемма доказана.

Теперь предложение 2 следует из теоремы Х. Еномото.

Из лемм 2.5 (2) и 3.1 следует, что граф Г, удовлетворяющий условиям предложения 1, не содержит пятиугольников. Предложение 1 вытекает из леммы 3.2 и предложения 2 .

\section{§4. Редукция $\mu$-регулярных графов без 3-лап}

В этом параграфее мы докажем следуюшее

ПРЕДЛОЖЕНИЕ 3. В связном $\mu$-регулярном графе Г без 3-лап либо все $\mu$-подграфы являются кликами, либо диаметр Г равен 2.

Графы диаметра 2, удовлетворяющие условиям предложения 3, описаны в работе [1].

ЛЕМма 4.1. Если $a, b$-смехсные вериины в графе Г без 3 -лап, то $[a]-b^{\perp}$ $u[b]-a^{\perp}$ являются кликами.

ДокАЗАтЕльство. Пусть $[a]-b^{\perp}$ содержит две несмежные вершины $c, d$. Тогда $b, c, d-3$-коклика из [a]. Лемма доказана. 
ДОКАЗАТЕЛЬСТВО ПРЕДЛОЖЕНИЯ 3. Пусть Г уДовЛетворяет условиям ПреД-

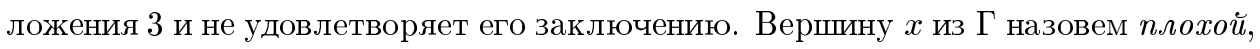
если $\Gamma_{3}(x)$ не пусто, в противном случае - хорошей. Поскольку $Г$ не удовлетворяет заключению предложения, то $\Gamma$ содержит плохие вершины. Вершину $x$ из $\Gamma$ назовем сильной, если $[x] \cap[y]$ содержит несмежные вершины для некоторого $y \in \Gamma_{2}(x)$, а пару $x, y$-сильной парой.

Лемма 4.2. Если $а, b$ - сильная пара вериин, а $c, d$-несмежнье верииньь из $[a] \cap[b]$, то $a^{\perp} \cup b^{\perp}=c^{\perp} \cup d^{\perp}$, и любая вериина из $\Gamma_{2}(a) \cap \Gamma_{2}(b)$ образует сильную пару с некоторой вершиной из $[a] \cap[b]$.

ДокАЗАтЕльство. Поскольку $[a]$ и $[b]$ не содержат 3 -коклик, то $[a] \cup[b] \subset$ $c^{\perp} \cup d^{\perp}$ и, значит, $a^{\perp} \cup b^{\perp}=c^{\perp} \cup d^{\perp}$. Отсюда $[b]-[a] \subset \Gamma_{2}(a)$.

Пусть $w \in \Gamma_{2}(a) \cap \Gamma_{2}(b)$ и $[x] \cap[w]-$ клика для любого $x \in\{a, b, c, d\}$. Положим $\Delta=([a] \cup[b]) \cap[w]$. Тогда $|\Delta|=2 \mu$.

Допустим сначала, что $[a] \cap \Delta \neq[c] \cap \Delta$. Пусть $[c]$ содержит $\alpha$ вершин из $[a] \cap \Delta$ и $\mu-\alpha$ вершин из $[b] \cap \Delta$ и $[d] \cap \Delta=\Delta-[c]$. Без ограничения обшности можно считать $\alpha \geqslant \mu / 2$.

Пусть $x \in[a] \cap[d] \cap \Delta, y \in[b] \cap[c] \cap \Delta$. Если вершины $x, y$ не смежны, то $[x] \cap[y]$ содержит $w$ и $2 \alpha$ вершин из $\Delta$. Противоречие. Значит, вершина $x$ смежна с $\mu$ вершинами из $[b] \cap \Delta$. Противоречие с тем, что $[b] \cap[x]$ содержит еше вершину $d$.

Пусть теперь $[a] \cap \Delta=[c] \cap \Delta$. Выберем вершину $x$ из $[a] \cap[w]$. Тогда $[x]$ содержит $\mu$ вершин из $[b]$, причем $[x]-a^{\perp}$ лежит в $w^{\perp}$. Далее, $[x]$ не содержит вершину $d$ из $[a] \cap[b]$, поэтому $[x]$ содержит некоторую вершину из $[b] \cap[w]$.

Пусть $[x]$ содержит $\alpha$ вершин из $[a] \cap[b]$. Тогда $[x]$ содержит $\mu-\alpha$ вершин из $[b] \cap[w]$. Заметим, что $x^{\perp} \cap a^{\perp}=x^{\perp} \cap c^{\perp}$, иначе $[x]$ содержит 3-коклику. Выберем вершину $y$ из $([b] \cap[w])-[x]$ и пусть $[y]$ содержит $\beta$ вершин из $[a] \cap[b]$. Тогда $[x] \cap[y]$ содержит $w, \mu-\alpha$ вершин из $[b] \cap[w]$ и $\mu-\beta$ вершин из $[a] \cap[w]$. Отсюда $(\mu-\alpha)+(\mu-\beta)<\mu$ и $\mu<\alpha+\beta$. Таким образом, $[x] \cap[y]$ содержит вершину $z$ из $[a] \cap[b]$, и $w, z-$ сильная пара.

ЛЕмма 4.3. Любая сильная вершина является хорошей.

ДокАЗАтЕльство. Достаточно доказать это утверждение для вершины $a$. Пусть $e \in \Gamma_{3}(a)$ и a fge-3-путь. Если $g \in[b]$, то $\mu$-подграф $[a] \cap[g]$ лежит в [b], иначе $[g]$ содержит $b, e$ и вершину из $([a] \cap[g])-[b]$, которые образуют 3 -коклику, что противоречит условиям предложения 3 . По лемме $4.2[g]-[a] \subset \Gamma_{2}(a)$. Противоречие. Значит, $[g]$ не содержит вершин, образующих сильную пару с вершиной $a$.

Если $g \in \Gamma_{2}(b)$, то по лемме $4.2 g$ образует сильную пару с некоторой вершиной $x \in[a] \cap[b]$. Пусть $y, z-$ несмежные вершины из $[x] \cap[g]$. Тогда $е$ смежна с одной из этих вершин, например с $y$. Тогда $g \in \Gamma_{2}(a) \cap[b]$. Заменив $g$ на вершину $y$, a $f$ на вершину из $[a] \cap[y]$ в 3 -пути $a f g e$, получим противоречие с утверждением предыдушего абзаца. Значит, $g \notin b^{\perp} \cup \Gamma_{2}(b)$.

По лемме $4.2 f \in[c] \cup[d]$. Пусть для определенности $f c$-ребро. Тогда мы получим путь $b c f g$ и $g \in \Gamma_{3}(b)$. Противоречие с тем, что $[f]$ содержит вершину $a$, которая образует сильную пару с $b$.

Таким образом, $\Gamma_{3}(a)$ пусто и каждая сильная вершина является хорошей. $\mathrm{B}$ частности, $\Gamma-\left(a^{\perp} \cup b^{\perp}\right)=\Gamma_{2}(a) \cap \Gamma_{2}(b)$. Лемма доказана.

Пусть $\Delta$ - множество плохих вершин, $a, b$ - фиксированная сильная пара. Если $\Delta$ содержит плохую вершину е из $[a] \cap[b]$, то $\Gamma_{3}(e) \subset \Gamma_{2}(a) \cap \Gamma_{2}(b)$. Противоречие с 
тем, что $\Gamma_{2}(a) \cap \Gamma_{2}(b)$ состоит из хороших вершин. Значит, $\Delta \subset([a]-[b]) \cup([b]-[a])$. Таким образом, если $e-$ плохая вершина, то она смежна с единственной вершиной из $\{a, b\}$ для любой сильной пары $a, b$.

Выберем плохую вершину е из $[a]-[b]$. Пусть $\Lambda=\Gamma-\left(a^{\perp} \cup b^{\perp}\right), c, d$-несмежные вершины из $[a] \cap[b]$. Для любых двух вершин $x, y$ на расстоянии 2 в $\Gamma$ через $\Lambda(x, y)$ обозначим подграф $\Gamma-\left(x^{\perp} \cup y^{\perp}\right)$. Фиксируем плохую вершину $e$ из $[a]-[b]$ и вершину $f$ из $\Gamma_{3}(e)$. Заметим, что ввиду леммы 4.2 любой $\mu$-подграф для вершины из пути длины 3 является кликой.

Лемма 4.4. Вершина $f$ принадлежит $[b]-[a]$, и $\Lambda$ содержится в $[e] \cup[f]$.

ДокАЗАтЕльство. Поскольку $e-$ плохая вершина, то $f \in[b]-[a]$. Далее, $e \in[c] \cup[d]$. Пусть $e$ не смежна с вершиной $y$ из $\Lambda$. По лемме $4.2 y$ образует сильную пару с некоторой вершиной $x$ из $[a] \cap[b]$. Так как $e \notin[y]$, то $е$ смежна с $x$. Отсюда $f \notin[x]$, поэтому $f$ смежна с $y$. Лемма доказана.

Заметим, что $e, f$ принадлежат $c^{\perp} \cup d^{\perp}$. Пусть, для определенности, $e \in c^{\perp}$. Тогда $f \in d^{\perp}$.

Лемма 4.5. Пусть $x, y$ - вершины из графа Г такие, что $d(x, y)=2$. Если $u \in[x]-[y]$, а $w$ - смежная с и вершина из $[y]-[x]$, то $[u] \cap \Lambda(x, y)=[w] \cap \Lambda(x, y)$.

ДокАЗАТЕльство. Так как $[u]-x^{\perp}$ является кликой, содержашей $w$, то $[u] \cap \Lambda(x, y) \subset[w]$. Аналогично, $[w] \cap \Lambda(x, y)$ содержится в $[u]$.

Лемма 4.6. Eсли $d(x, y)=2$, то $\Lambda(x, y)$ - клика из $k-2 \mu+1$ вершин, совпадающая $c \Gamma_{2}(x) \cap \Gamma_{2}(y)$.

ДокаЗАтЕльство. Заметим, что $[e]$ содержит вершину $g$ из $[b]-[a]$, иначе $[e] \cap[b] \subset[a]$ и $e-$ хорошая вершина. Ясно, что $[f] \cap[g]-$ клика из $b^{\perp}-[a]$, так как $a \in \Lambda(f, g)$ и по лемме $4.5[e] \cap \Lambda=[g] \cap \Lambda$. Теперь $[b] \subset f^{\perp} \cup g^{\perp}$, поэтому $\Lambda(f, g)$ содержится в $a^{\perp}$ и состоит из $k-2 \mu+1$ вершин.

Если $y \in \Lambda$, то $[y]$ содержит не более $2 \mu$ вершин из $f^{\perp} \cup g^{\perp}$. Отсюда $y \in$ $\Gamma_{2}(f) \cap \Gamma_{2}(g)$ и $[y]$ содержит $k-2 \mu$ вершин из $\Lambda(f, g)$. Таким образом, $\Lambda(f, g)$ - клика. Так как $|\Lambda(x, y)|=k-2 \mu+1$ для любых двух вершин на расстоянии 2 , то последнее рассуждение можно повторить для любой такой пары вершин $x, y$.

ЛЕмма 4.7. Справедливы соотношения $\Gamma=e^{\perp} \cup f^{\perp},|\Gamma|=6 \mu,|k|=3 \mu-1$ $u|\Lambda|=\mu$.

ДокАЗАТЕЛЬСтво. По лемме $4.6|\Gamma|=3(k-\mu+1)$. С другой стороны, если $x \in \Gamma-\left(e^{\perp} \cup f^{\perp}\right)$, то $|\Gamma| \geqslant 2(k+1)+k-2 \mu+1$. Но тогда $\mu=0$, противоречие. Значит, $|\Gamma|=2(k+1)$ и $k=3 \mu-1$. Лемма доказана.

Вершину $x$ назовем 0-точкой для $\Lambda$, если $|[x] \cap \Lambda|=0$.

ЛЕмма 4.8. Вериины е и $f$ не могут бъть 0-точками для подграфа $\Lambda$.

ДокаЗАТЕЛЬСтво. Пусть $e-0$-точка для $\Lambda$. Тогда $\Lambda(e, b)=\Lambda$ и $e^{\perp}$ содержит $[a]-[b]$. В этом случае $[f]$ не содержит вершин из $[a]-[b]$ и $[a] \cap[f]=[a] \cap[b]$, противоречие с леммой 4.3. Лемма доказана.

Зафиксируем вершины $u \in[e] \cap \Lambda, w \in[f] \cap \Lambda$. 
Лемма 4.9. Подграф $\Lambda(u, b)$ содержит все вершины из $([a]-[b]) \cap[f] и$ лежит в $[d] \cap a^{\perp}$.

ДокАЗАТЕЛЬСТво. Ввиду леммы $4.6 \Lambda(u, b)$ содержится в $a^{\perp}-[b]$. Если $h-$ вершина из $([a]-[b]) \cap[f]$, смежная с $u$, то $[h]$ содержит 3-коклику $\{a, f, u\}$, что противоречит условию теоремы $1 . \Lambda(u, b) \cap[f]$ содержится в $[d]$, так как $[a] \cap[f]$ - клика из $d^{\perp}$. Пусть $\Lambda(u, b)-[f]$ содержит вершину $x$, не смежную с $d$. Тогда $x \in[c]-[d]$. Если $y \in[f] \cap([a]-[b])$, то $x y$ - ребро, так как $\Lambda(u, b)-$ клика. $\mathrm{C}$ другой стороны, $[c] \cap[f]-$ клика из $b^{\perp}$, поэтому $y \in[d]-[c]$. По лемме 4.5, примененной к $\Lambda=\Lambda(c, d)$ и к $\Lambda(u, b),[x] \cap \Lambda=[y] \cap \Lambda$ и $[f] \cap \Lambda(u, b)=[w] \cap \Lambda(u, b)$, поэтому $w \in[x]$. Противоречие с выбором $x$. Лемма доказана.

Из симметричности следует, что подграф $\Lambda(w, c)$ содержит все вершины из $([d]-[c]) \cap[e]$ и лежит в $[a] \cap d^{\perp}$. Аналогично, подграф $\Lambda(u, d)$ содержит все вершины из $([c]-[d]) \cap[f]$ и лежит в $[b] \cap c^{\perp}$. Подграф $\Lambda(w, a)$ содержит все вершины из $([b]-[a]) \cap[e]$ и лежит в $[c] \cap b^{\perp}$.

Лемма 4.10. Справедливы следующие утверждения:

(1) $\Lambda(u, b) \cup \Lambda(w, c)=([a] \cap[f]) \cup([d] \cap[e])$;

(2) $\Lambda(u, d) \cup \Lambda(w, a)=([b] \cap[e]) \cup([c] \cap[f])$.

ДокАЗАТЕЛЬСтво. Докажем только первое равенство. Заметим, что включение слева направо следует из леммы 4.9. Покажем, что $\Lambda(u, b)$ не пересекает $\Lambda(w, c)$. По лемме $4.5[f] \cap \Lambda(u, b)=[w] \cap \Lambda(u, b)$, поэтому $[f] \cap \Lambda(u, b)$ не пересекает $\Lambda(w, c)$. Аналогично, $[e] \cap \Lambda(w, c)=[u] \cap \Lambda(w, c)$. Лемма доказана.

Рассмотрим теперь подграф̆ $\Sigma$, где

$$
\Sigma=\bigcup_{(x, y) \in A} \Lambda(x, y), \quad A=\{(u, b),(u, d),(w, a),(w, c)\}
$$

Лемма 4.11. Подараф $\Sigma$ состоит из $4 \mu$ вериин.

ДокАЗАТЕЛЬСтво. Напомним, что по лемме $4.7 \quad \Lambda(u, b)$ содержит $\mu$ вершин. Поскольку все четыре подграфа, которые составляют $\Sigma$, симметрично расположены в графе $\Gamma$, то для доказательства леммы достаточно показать, что подграф $\Lambda(u, b)$ не пересекает остальные подграфы $\Lambda(x, y)$. Но $\Lambda(u, b)$ не пересекает $\Lambda(u, d)$ по лемме 4.9 , а $\Lambda(w, c)$ по лемме 4.10. Далее, $\Lambda(u, b) \cap[f]$ содержится в $[w]$, так как $[b]-f^{\perp}$ клика из $[w]$. Аналогично, $\Lambda(w, a) \cap[e]$ содержится в $[u]$, поэтому $\Lambda(u, b)$ не пересекает $\Lambda(w, a)$. Лемма доказана.

Завершим теперь доказательство предложения 3. Для этого выберем вершину $x$ из множества $\{e, f\}$ так, что $|[x] \cap \Sigma| \geqslant 2 \mu$. Пусть для определенности $x=f$. Покажем, что $\mu$-подг раф $[u] \cap[f]$ не пересекает $\Sigma$. Напомним, что $[u] \cap[f]-$ клика, содержашая $\Lambda \cap[f]$, поэтому подграф $[u] \cap[f]$ не пересекает $\Lambda(w, a)$ и $\Lambda(w, c)$. Далее, $[u] \cap[f]$ не пересекает $\Lambda(u, b)$ и $\Lambda(u, d)$, по определению. Итак, $\mu$-подграф $[u] \cap[f]$ не пересекает $\Sigma$. Однако по леммам 4.7 и 4.11 подграф $[f]-\Sigma$ содержит не более $\mu-1$ вершин. Противоречие. Предложение 3 доказано. 


\section{§5. Вполне регулярные графы Тервиллигера без 3-лап}

Пусть $Г$ - связный вполне регулярный граф Тервиллигера с параметрами $(v, k, \lambda, \mu), \mu>1$. Подграф $\Delta$ из графа $\Gamma$ называется сингулярной прямой, если для любых различных $a, b$ из $\Delta$ верно равенство $\Delta=\left\{x \in \Gamma \mid x^{\perp} \supset a^{\perp} \cap b^{\perp}\right\}$.

По теореме Тервиллигера [3] (см. также [4, теорема 1.16.3]) для любой вершины $a$ графа $\Gamma$ редуцированный граф для $[a]$ является сильно регулярным графом Тервиллигера с параметрами

$$
\begin{gathered}
\bar{v}=\frac{k}{s}, \quad \bar{k}=\frac{\lambda-s+1}{s}, \quad \bar{\mu}=\frac{\mu-1}{s}, \\
\bar{\lambda}=\frac{(\lambda-s+1)(\lambda-2 s+1)-(\mu-1)(k-\lambda-1)}{s(\lambda-s+1)},
\end{gathered}
$$

где $s$ - число вершин в классе эквивалентности для отношения $\equiv$, где $x \equiv y$, если $x^{\perp}=y^{\perp}$ (редуцированный граф - это фактор-граф по отношению эквивалентности 三). Далее, каждая сингулярная прямая имеет $s+1$ вершину, и каждая вершина лежит в $k / s$ сингулярных прямых. В частности, число $s$ делит наибольший общий делитель для чисел $k, \lambda+1, \mu-1$, а число $\lambda-s+1$ делит $(\mu-1)(k-s)$.

ПРЕДЛОЖЕНИЕ 4. Пусть Г - связный реберно регулярный граф без 3-лап с параметрами $(v, k, \lambda)$, в котором все $\mu$-подграфы являются кликами. Тогда Г - вполне регулярный граф Тервиллигера и либо $\mu=1$, либо Г - граф икосаәдра с параметрами $(12,5,2,2)$.

Заметим, что попытка получения $\mu$-регулярности в произвольном реберно регулярном графе без 3-лап наталкивается на контрпример (см. рис. 2 во введении).

Пусть граф Г удовлетворяет условиям предложения 4.

ЛЕмма 5.1. ГрафГ - вполне регулярен с $\mu=2 \lambda+3-k$.

ДокАЗАТЕЛЬСтво. Пусть $d(a, b)=2, c \in[a] \cap[b]$ и $\mu=|[a] \cap[b]|$. Тогда по лемме $4.1[c]$ содержится в $a^{\perp} \cup b^{\perp}$, поэтому $k=2(\lambda+1)-(\mu-1)$. Итак, $\mu=2 \lambda+3-k$ и, следовательно, не зависит от выбора вершин $a, b$. Лемма доказана.

Пусть далее $\mu>1$.

Лемма 5.2. Пусть $d(a, b)=2, c \in[a] \cap[b]$. Тогда сингулярная прямая, которая проходит через вершинь $\{a, c\}$, пересекает $\mu$-подграф $[a] \cap[b]$ в единственной точке.

ДокАЗАТЕльство. Пусть различные вершины $c, d$ лежат на сингулярной прямой из $a^{\perp}$. Тогда $c^{\perp} \cap d^{\perp}$ содержит $a^{\perp} \cap c^{\perp}$ и вершину $b$. Противоречие с реберной регулярностью граф̆а.

Лемма 5.3. Пусть $a c-p e б р о, ~ b \in[c]-[a]$. Тогда для любой отличной от $b$ вершины е из $[c]-[a] \quad \mu-п о д г р а ф[a] \cap[e]$ содержит единственную вериину с из $[a] \cap[b]$.

ДокАЗАтЕльство. Предположим, что подграфф $[a] \cap[b] \cap[e]$ содержит $\alpha+1$ вершину, $\alpha>0$. Пусть $f$ отличная от $c$ вершина из этого подграфа. Тогда $f$ не смежна с некоторой вершиной $g$ из $([a] \cap[c])-([b] \cup[e])$, и $\mu$-подграф $[f] \cap[g]$ содержит $2(\mu-\alpha-1)$ вершин из $\mu$-подграфов $[a] \cap[b]$ и $[a] \cap[e]$, две вершины $a, c$ и $(\alpha+1)(s-1)$ 
вершин из сингулярных прямых, проходяших через $a$ и вершину из $[a] \cap[b] \cap[e]$. Отсюда $\mu+\alpha(s-3)+s-1 \leqslant 0$. Но тогда $s=1$. Поскольку любой отличный от пятиугольника граф Мура содержит 3-лапу [4, теорема 6.7.1], то Г - локально пятиугольный граф. Очевидно, что в этом случае заключение леммы выполняется.

Лемма 5.4. Параметр s равен $\lambda-2 \mu+3$.

ДокАЗАТЕЛЬСтво. Пусть $b, e-$ вершины из графа $\Gamma_{2}(a)$. По предыдушей лемме $\mu$-подграфы $[a] \cap[b]$ и $[a] \cap[e]$ пересекаются не более чем по одной вершине. Пусть $c \in[a] \cap[b]$. Если $[a] \cap[b]=[a] \cap[e]$ для любой вершины $e$ из $[c]-a^{\perp}$, то весь $\mu$-подграф $[a] \cap[b]$ лежит в $K(a)$, что противоречит реберной регулярности графа $\Gamma$. Значит, $[a] \cap[b]$ и $[a] \cap[e]$ пересекаются в точности по вершине $c$. Ввиду леммы 4.1 $([a] \cap[c])-[b]$ и $([a] \cap[c])-[e]$ клики, поэтому все вершины из $([a] \cap[c])-([e] \cup[b])$ лежат на сингулярной прямой, проходящей через $\{a, c\}$. Значит, $s=\lambda-2 \mu+3$. Лемма доказана.

ЛЕмма 5.5. Граф Г с $s=\lambda-2 \mu+3$ является графом икосаэдра.

ДоказАтЕльство. По лемме $5.1 k=2 \lambda-\mu+3$. По теореме Тервиллигера и из равенств $\mu=s \bar{\mu}+1, \lambda=s+2 s \bar{\mu}-1$ и $k=2 s+3 s \bar{\mu}$ мы имеем $\bar{k}=2 \bar{\mu}$, $\bar{\lambda}=\frac{3}{2}(\bar{\mu}-1)$. Если параметры редуцированного графа для $[a]$ не удовлетворяют половинному случаю, то число $(\bar{\lambda}-\bar{\mu})^{2}+4(\bar{k}-\bar{\mu})$ является квадратом, поскольку этот граф сильно регулярен. Подставляя $\bar{k}$ и $\bar{\lambda}$ в это выражение, мы получим $\frac{1}{4}\left(\bar{\mu}^{2}+10 \bar{\mu}+9\right)$. Однако, $\bar{\mu}^{2}+10 \bar{\mu}+9$ меньше, чем $(\bar{\mu}+5)^{2}$ и больше, чем $(\bar{\mu}+3)^{2}$. Значит, $\bar{\mu}^{2}+10 \bar{\mu}+9=(\bar{\mu}+4)^{2}$. Противоречие с тем, что это уравнение не имеет целых корней. Если параметры редуцированного графа для $[a]$ удовлетворяют половинному случаю, то $\bar{\mu}=1$ и редуцированный граф̆ для $[a]$ является пятиугольником. В этом случае $\mu=s+1, \lambda=3 s-1$ и $k=5 s$. Однако, по $\mu$-подграфу $[a] \cap[b]$ строится клика $\Sigma$ порядка $2 \mu s-\mu$ следуюшим образом: для любой вершины $c$ из $[a] \cap[b]$ в $\Sigma$ включаем сингулярные прямые, проходящие через $a, c$ и $b, c$. Если теперь $c, d$ различные вершины из $[a] \cap[b]$, то $c^{\perp} \cap d^{\perp}$ содержит $\Sigma \cup\{a, b\}$. Отсюда $\lambda \geqslant 2 \mu s-\mu$. Значит, $3 s-1 \geqslant 2 s^{2}+s-1, s=1$, и снова $\Gamma$ - локально пятиугольньй граф. По предложению 1.1.4 из [4] связный локально пятиугольный граф является графом икосаэдра. Лемма и предложение 4 доказаны.

Объединяя результаты $\S \S 3,4$ и 5 , получаем теорему 2.

\section{§6. Редукция регулярных графов Тервиллигера без 3-лап}

В этом параграфе мы докажем

ПРеДЛОЖЕнИЕ 5. Связный регулярный граф Тервиллигера без 3-лап является $\alpha$-расширением графа с $\mu=1$ или графа икосаэдра.

Граф усеченного тетраэдра (рис. 1 во введении) показывает, что регулярный граф̆ Тервиллигера с $\mu=1$ не обязательно реберно регулярен.

Следуюшее утверждение справедливо для произвольных графов Тервиллигеpa.

Лемма 6.1. Пусть Г - связный регулярный граф Тервиллигера, а - вершина из Г. Тогда для любого ребра $b c$ из $[a]-K(a)$ найдется вершина $d$, принадлежащая $a^{\perp}$ инесмежная $c$ b и 
ДокАЗАТЕЛьство. Воспроизведем рассуждения из пункта (2) доказательства леммы 3.4 работы [3]. Пусть $a^{\perp}$ содержится в $b^{\perp} \cup c^{\perp}$. По выбору $b$ и $c$ разность $[b]-c^{\perp}$ содержит вершину $x$ из $[a],[c]-b^{\perp}$ содержит $y$ из $[a]$. Заметим, что вершины $x$ и $y$ не смежны, иначе $[x] \cap[c]$ содержит несмежные вершины $b$ и $y$.

Теперь разность $([x] \cap[y])-K(a)$ содержится в $[b] \cap[c]$. В самом деле, если $z$ - несмежная с $b$ вершина из $[x] \cap[y]$, то $z$ смежна с $c$ по предположению, и $[b] \cap[z]$ содержит несмежные вершины $x$ и $c$. Теперь $[y] \cap[b]$ содержит весь $\mu$-граф $[x] \cap[y]$ и вершину $c$. Противоречие. Лемма доказана.

Пусть теперь $Г$ - контрпример к предложению 5 и параметры Г равны $(v, k, \mu)$.

ЛЕмма 6.2. Если $a, b$ - несмежсные вериины из $[c], \lambda_{1}=|[a] \cap[c]|, \lambda_{2}=$ $|[b] \cap[c]|$, mo $\lambda_{1}+\lambda_{2}=k+\mu-3$.

ДокАЗАтЕльство. Поскольку граф $Г$ не содержит 3 -лап, то $[c] \subset a^{\perp} \cup b^{\perp}$. Поэтому $k=\left(\lambda_{1}+\lambda_{2}\right)-(\mu-1)+2$.

ЛЕмма 6.3. Если ас-ребро из $\Gamma, a \notin K(c),|[a] \cap[c]|=\frac{1}{2}(k+\mu-3)$, то для любого ребра $x y, x \notin K(y)$, верно равенство $|[x] \cap[y]|=\frac{1}{2}(k+\mu-3)$.

ДокАЗАТЕЛьство. Ввиду связности графа Г достаточно доказать требуемое равенство для ребра $c b$. Если $b \notin a^{\perp}$, то требуемое равенство следует из леммы 6.2. Если $b \in a^{\perp}$, то по лемме $6.1[c]$ содержит вершину $d$, несмежную с $a$ и $b$. Теперь можно применить предыдушее рассуждение к парам $a, d$ и $b, d$. Лемма доказана.

По выбору графа Г найдутся такие вершины $a, b$ на расстоянии 2 в $\Gamma$, что $\mu$-граф $[a] \cap[b] \neq K(c)$ для некоторой вершины $c$ из $[a] \cap[b]$. Положим $\lambda=\frac{1}{2}(k+\mu-3)$.

Лемма 6.4. Для любой вершины $x$ из $c^{\perp}-K(c)$ верно равенство $|[x] \cap[c]|=\lambda$.

ДокАЗАТЕЛЬСтво. Положим $\lambda_{1}=|[a] \cap[c]|, \lambda_{2}=|[b] \cap[c]|$ и выберем $d$ из $([a] \cap[b])-K(c)$. По лемме $6.2 \lambda_{1}+\lambda_{2}=k+\mu-3$. По лемме $6.1[c]$ содержит вершину $x$, несмежную с $a$ и $d$. Тогда $|[x] \cap[c]|=\lambda_{2}$ и, следовательно, $|[c] \cap[d]|=\lambda_{1}$.

Симметрично, $[c]$ содержит вершину $y$, несмежную с $b$ и $d$. Повторив рассуждения из предыдущего абзаца, получим $|[c] \cap[d]|=\lambda_{2}$. Теперь утверждение леммы следует из леммы 6.3. Лемма доказана.

Положим $\Lambda=c^{\perp}-K(c)$ и $\alpha=|K(c)|$.

ЛЕмма 6.5. Все классы әквивалентности $\bar{x}$ в графе $\Lambda$ имеют одинаковую мощность $s$, редуцированный граф $\bar{\Lambda}$ является пятиугольником. Более того, $\alpha=2 \mu-k, 4 s=k-\mu+1 u \lambda=2 s+\alpha-2$.

ДокАЗАТЕльство. По лемме 6.4 и предложению 1.16 .2 из [4] все классы эквивалентности $\bar{x}$ для $x \in \Lambda$ имеют одинаковую мощность $s$ и редуцированный граф $\bar{\Lambda}$ является сильно регулярным графом Тервиллигера с параметрами $(\bar{v}, \bar{k}, \bar{\lambda}, \bar{\mu})$, где

$$
\bar{v}=\frac{k-\alpha+1}{s}, \quad \bar{k}=\frac{\lambda+2-\alpha-s}{s}, \quad \bar{\mu}=\frac{\mu-\alpha}{s},
$$

и $\bar{\lambda}$ вычисляется из прямоугольного соотношения. Ввиду предложения 4 параметр $\bar{\mu}$ равен 1. Следовательно, окрестность любой вершины в $\Lambda$ расщепляется двумя кликами порядка $\bar{\lambda}+1$. Легко видеть, что в этом случае $\bar{\lambda}=0$ (см., например, [4, теорема 1.2.3]). Теперь граф $\bar{\Lambda}$ является пятиугольником и $\mu=\alpha+s$, $\lambda=2 s+\alpha-2, k=5 s+\alpha-1$. Лемма доказана. 
ЛЕмма 6.6. Ядро $K(x)$ состоит из $\alpha$ вершин для любой вершины $x$ из графа $\Gamma$.

ДокаЗАтЕЛЬство. Если $|K(x)|<\mu$, то к графу $x^{\perp}-K(x)$ применяем лемму 6.5 и получаем $|K(x)|=\alpha$.

Пусть теперь $|K(x)|=\mu$. Выберем путь $c=w_{0}, w_{1}, \ldots, w_{n}=x$ и пусть $i-$ наименьший номер такой, что $\left|K\left(w_{i}\right)\right|=\mu$. Тогда $w_{i-1}^{\perp} \cap w_{i}^{\perp}$ является кликой порядка $\lambda+2$ из $w_{i}^{\perp}$. По лемме 6.5 , примененной к $w_{i-1}^{\perp}-K\left(w_{i-1}\right)$, получаем $\lambda+2=s+\alpha=2 s+\alpha$. Противоречие. Лемма доказана.

По леммам 6.5 и 6.6 редуцированный граф $\bar{\Gamma}$ является вполне регулярным графом, и предложение 5 следует из предложения 4. Теперь теорема 1 вытекает из предложений 3,5 и работы [1].

\section{Список литературы}

1. Кабанов B. В., Махнев A. А. Кореберно регулярные грабы, в которых антиокрестности вершин кореберно регулярны // III Международная конференция по алгебре. Тез. докл. Красноярск, 1993. С. 139; Кореберно регулярные графы без 3-лап. Т. 60. №4, 1996. C. 495-503.

2. Enomoto $H$. Characterization of families of finite permutation groups by subdegrees. II // J. Fac. Sci. Univ. Tokyo Sect. IA Math. 1973. V. 20. P. 1-11.

3. Terwilliger P. Distance-regular graphs with girth 3 or 4, I // J. Combin. Theory Ser. B. 1985. V. 39. P. 265-281.

4. Brouwer A.E., Cohen A.M., Neumaier A. Distance-Regular Graphs. Springer-Verlag, 1989. 